\title{
Editorial
}

\section{Advances in biomedical engineering: a call for enhancing empirical research}

\author{
Andrés Orozco-Duque ${ }^{1}$ and Daniel Novák ${ }^{2}$
}

${ }^{1}$ Grupo de Investigación e Innovación Biomédica, Facultad de Ciencias Exactas y Aplicadas, Instituto Tecnológico Metropolitano, Medellín, Colombia

${ }^{2}$ Department of Cybernetics, Faculty of Electrical Engineering, Czech Technical University in Prague, Czech Republic

Advances in biomedical engineering (BME) imply the existence of research groups working in multidisciplinary teams to understand physiological processes and develop methods and tools for diagnostics and therapeutics. Multidisciplinary teams include physicians, biologists, physicists, mathematicians and engineers from different disciplines: electrical and electronics, computer sciences, materials, mechanical, chemical, among others. Lately, BME has become a bridge joining these disciplines. Therefore, successful BME projects involve not only a deep knowledge of the specific discipline, but also an understanding of the physiological phenomena under study.

The overall objectives of this special issue on advances in biomedical engineering are (1) to present the current state of the art of nanotechnology and the Internet of Things (IoT); (2) to advance understanding of how robots can solve challenging problems - e.g., brain-shift during neurosurgery; and (3) to present original research articles about the application of modeling, simulation and signal processing in biomedical engineering. This issue also explores future opportunities of using simulation techniques, particularly in clinical practice, and discusses challenges and limitations of computer simulations.
Particularly, for this special issue on advances in biomedical engineering five manuscripts were selected. The first contribution aligns ongoing IoT integration and the emerging nano-tech approach. Nowadays, mobile phones, tablets and smart watches are becoming popular platforms that are affordable and powerful enough for the majority of IoT applications. Furthermore, we observe high adoption rates in the emerging market of the Internet of Things along with persuasive gadgets. Many physiological parameters are seamlessly integrated into mobile wearables such as heart rate monitors, pulse oximeters, blood pressure cuffs or glucometers. All these developments combined provide an environment that facilitates effective and timely delivery of medical solutions that are sophisticated and relevant. Furthermore, this advance in macro IoT concepts provides a strong foundation to micro and nanotechnological platforms.

The second contribution copes with the difficult task of compensating for brain shift, a common source of noise during neurosurgical procedures. To obtain accurate location information about surgical targets in neuro-surgery, a frequently employed method is called microrecording exploration. The recorded signals are concurrently evaluated by a trained neurologist who 
then identifies the optimal position for the stimulation contacts. The evaluation is typically based on visual and auditory inspection of the signals and the main markers are neuronal firing patterns, especially the amplitude of the neuronal background, which is higher in areas with higher neuronal density —where the target is supposed to be located [1]. This process could be naturally replaced by mathematical modelling that creates space for automatic brain-shift corrections.

The third and fourth manuscripts describe the challenging task of cardiovascular tissue modelling. The process for identifying correct ablation sites has been studied in recent years and several methods, measures and approaches have been described in atrial fibrillation literature. Thus, the third article addresses the important issue of intracardiac electrograms analysis, for ablation sites location in AF, using a novel framework of time-singularity multifractal spectrum distribution. Furthermore, the fourth work looks into the effect of air pollutants on human atrial myocytes using computational models.

The last contribution sheds light on post-traumatic syndrome in connection with emotional processing. The objective of this work is to define brain connectivity to identify differences in emotional processing between excombatants and individuals not directly exposed to the armed conflict.

Research studies in BME mainly involve applied science research as well as Design and Development projects (D\&D). The latter include Modeling and Simulation (M\&S), which is nowadays an integral part of the scientific process [2]. According to Ellis and Levy, research in $\mathrm{D} \& \mathrm{D}$ is defined as "addressing an acknowledged problem, building upon existing literature, and making an original contribution to the body of knowledge" [3]. Therefore, research in $\mathrm{D} \& \mathrm{D}$ is not only limited to product development, but also knowledge of previous results and validation as key factors. Thus, empirical research must be conducted in this regard [4][5].

Basic and applied science usually conduct experiments according to the requirements of the scientific method. Medical science has a rich history of empirical research (although there are still problems) [6]. However, there are drawbacks in a number of D\&D projects: the lack or weakness in the use of empirical research methods. For instance, the editorial board of BioMedical Engineering OnLine claims that many papers "lack sufficiently clear description of the validation methods to enable readers to judge the validity of the work" [7]. For now, we want to emphasize some cautions to consider when we conduct empirical research in D\&D:

- Researchers must know previous research results in detail, both in their particular field of knowledge and in the multidisciplinary fields involved in the study problem.

- Study results must be replicable, and relevant previous studies must be used as reference for results comparison.

- Authors should emphasize on the medical application. According to Sjober et al., some studies "are merely demonstrations that a technology works ('proof of concept') or simple experience reports ('lessons learned') [8]."

- Special care must be taken to avoid generalizations without statistical basis or without enough description of the limitations and assumptions.

To enhance the empirical method in D\&D projects, post-graduate engineering programs (Master's and Ph.D.) should 
strengthen the guidance and instruction on the scientific method and empirical research. Likewise, all members of the scientific and academic community in our role as students, tutors, authors or peer-reviewers should aim to contribute to the improvement of conducting and reporting research in $\mathrm{BME}$.

\section{REFERENCES}

[1] E. Bakštein et al., "Methods for automatic detection of artifacts in microelectrode recordings," $J$. Neurosci. Methods, vol. 290, pp. 39-51, 2017.

[2] S. RM, "The scientific method is dead--long live the (new) scientific method," Surg Innov., vol. 12, no. 2, pp. 173-6, 2005.

[3] T. J. Ellis and Y. Levy, "A Guide for Novice Researchers: Design and Development Research Methods," Proc. Informing Sci. IT Educ. Conf., no. 10, pp. 107-118, 2010 .
[4] A. J. Ko, T. D. LaToza, and M. M. Burnett, "A practical guide to controlled experiments of software engineering tools with human participants," Empir. Softw. Eng., vol. 20, no. 1, pp. 110-141, 2015.

[5] B. A. Kitchenham and T. Dybå, "Evidence-based Software Engineering," 2004.

[6] R. J. Stusser, "Reflections on the Scientific Method in Medicine," in Medical and Health Sciences Volume VIII -, 2010, pp. 53-77.

[7] K. R. Foster, R. Koprowski, and J. D. Skufca, "Machine learning, medical diagnosis, and biomedical engineering research commentary," Biomed. Eng. Online, vol. 13, no. 1, p. 94, 2014.

[8] D. I. K. Sjøberg, T. Dybå, and M. Jørgensen, "The Future of Empirical Methods in Software Engineering Research," Futur. Softw. Eng. FOSE 07, no. 1325, pp. 358-378, 2007. 